\title{
'Nothing new': responses to the introduction of antiretroviral drugs in South Africa
}

Mickey Chopra ${ }^{a}$ b, Carl Kendallc, Zelee Hillc, Nikki Schaayb, Lungiswa L. Nkonkia and Tanya M. Dohertya,d

Interviews conducted in South Africa found that awareness of antiretroviral therapy was generally poor. Antiretroviral drugs were not perceived as new, but one of many alternative therapies for HIV/AIDS. Respondents had more detailed knowledge of indications, effects and how to access alternative treatments, which is bolstered by the active promotion and legitimization of alternative treatments. Many expressed a lack of excitement about the introduction of antiretroviral therapy, and little change in their attitudes concerning the epidemic.

The introduction of HIV treatment in developed countries has been credited with reducing the denial, stigma and discrimination of HIV/AIDS as a disease [1]. In South Africa the introduction of antiretroviral drugs seems to have had a limited effect on high levels of stigma [2], risk behaviour [3] and HIV incidence [4]. We set out to examine the potential cause of this lack of a wider effect especially with respect to the influence of other treatment options.

We conducted a total of 197 interviews across three districts where antiretroviral therapy had been introduced for at least 6 months. Interviews were conducted in three populations: 76 individuals on antiretroviral therapy, $58 \mathrm{HIV}$-positive women not taking antiretroviral drugs, 45 community members, and 18 community gate-keepers such as leaders of non-governmental organizations, health workers and representatives from local government.

Respondents were interviewed using pretested semi-structured guides. Transcripts were reviewed daily by site supervisors to identify emerging themes and ensure quality. Interviews were analysed through the systematic identification and coding of themes. The results were then discussed and consensus was reached in two analysis workshops by all authors. Ethical approval was granted by the University of the Western Cape and Tulane University.

Only seven of the 33 community respondents who had heard of antiretroviral agents reported them as a factor influencing the lifespan of a person with HIV, and only 10 reported antiretroviral treatment as a benefit of having an HIV test. Some explicitly expressed a lack of excitement about antiretroviral therapy: 'When I asked if he had heard of the new HIV medication he looked excited and said "no". ... As soon as I mentioned the word ARVs he said "oh those" and seemed to lose interest. ... I asked if people were excited about the provision of ARVs, he said "no they are nothing new, because they have said they are not curing, it's still the same".' (Community leader). A lack of excitement was also expressed by health workers: 'They (nurses in community clinics) just see it as another thing they have to 
do.' (Antiretroviral therapy nurse). Excitement was higher among the HIV-positive respondents: they reported that being on antiretroviral drugs had improved their health and given them hope, and nearly all of the 28 HIV-positive women who had heard of antiretroviral treatment reported that they would like to be on therapy at some point; however, only eight of the women had tried or were in the process of trying to access treatment.

All respondent groups reported that antiretroviral drugs were not a new class of treatment but, along with 21 other mass-produced 'immune boosters' also listed, were classified as boosting a person's 'body soldiers' (immune system); antiretroviral agents were, however, seen as particularly strong and powerful. Almost half (40\%) of the antiretroviral therapy respondent group reported that they had used one or more of the alternative treatments before starting antiretroviral therapy.

Alternative treatments were popular despite their cost, which, for some respondents, consumed a large proportion of their income. We found several reasons why alternative treatment was more attractive. Many of the alternative treatments have been available for many years and knowledge of them is much greater than knowledge of antiretroviral drugs as regards details such as where to access them and how to take them.

Manufacturers and sellers of alternative treatments have adopted strong and successful marketing strategies, whereas antiretroviral therapy awareness activities are relatively low key and are focused on providing factual information rather than on a hard sell (Table 1). Alternative treatments have been legitimized through several routes (Table 1), and there is a strong belief that they are efficacious. Because many of the alternative treatments are not specifically targeted towards HIV they can be used without disclosure or stigmatization, and before formal testing.

Finally, alternative treatments are widely distributed, do not require a long wait or a prescription, and can be accessed regardless of the stage of the illness. Antiretroviral drugs were available free of charge in all sites but there is a lengthy enrolment process. Antiretroviral drugs were perceived as being only for the critically ill, and the HIV- positive respondents reported using alternative treatments when they (or health staff) did not consider themselves sick enough for antiretroviral drugs or had difficulty accessing them: 'He asked the nurse about ARVs. ... The nurse said he is still strong and he can survive and advised him to seek traditional medicines. He went to a traditional healer that he had heard about in the community. ... He paid R5OO and lived at the traditional healer's for some time so that he could take the medicines well.' (Antiretroviral therapy respondent). 


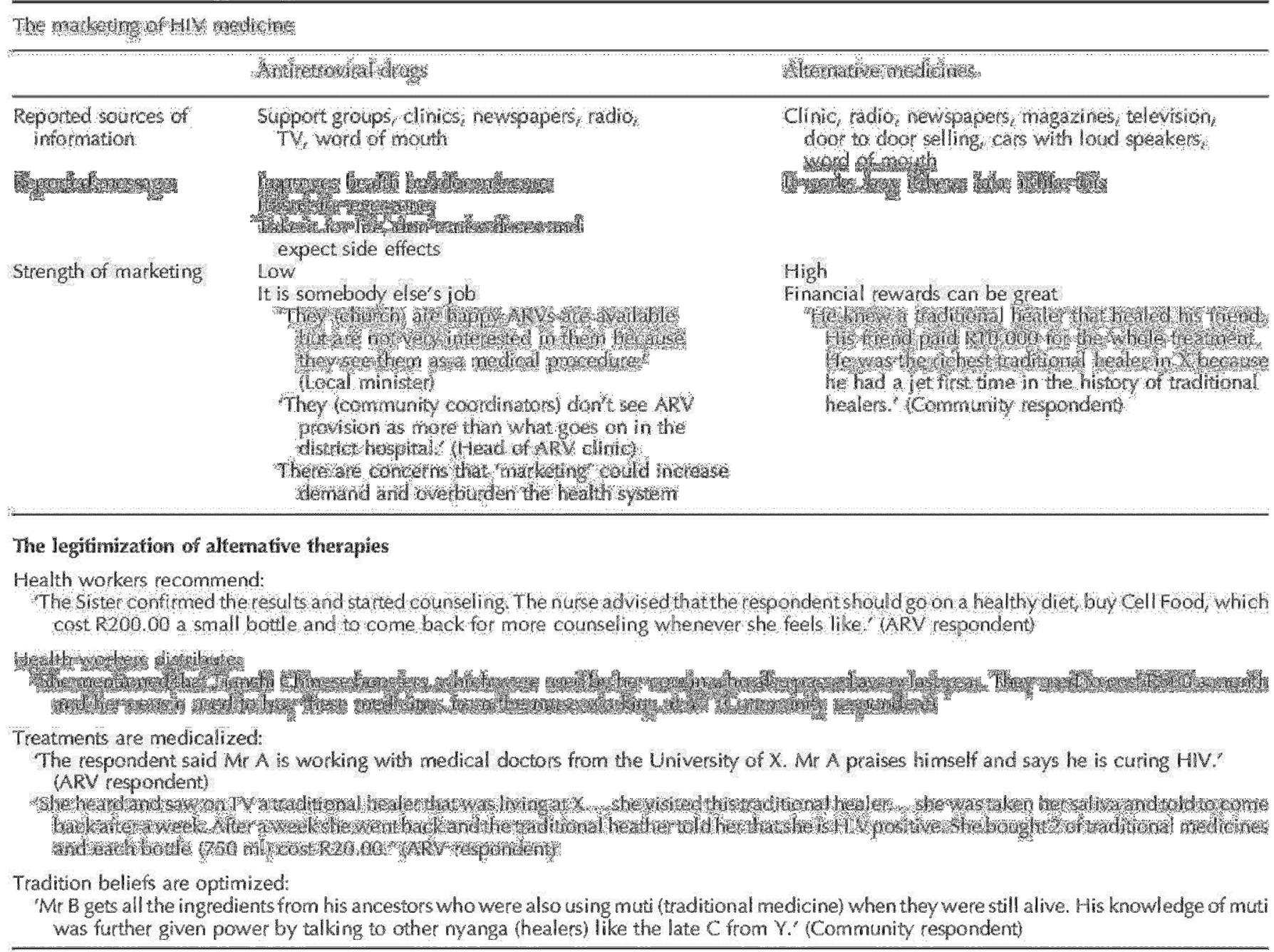

Antiretroviral therapies are being introduced into a complex HIV treatment environment where they are competing with a multitude of alternative treatments. The choices are overwhelming and range from age old indigenous 'cure-alls' to new herbal 'boosters'. Respondents across all three sites had more detailed knowledge of the indications, effects and how to access these alternative treatments than they did of antiretroviral drugs. This is being bolstered by the promotion and legitimization of alternative treatments and the contrasting lack of similar activities for antiretroviral therapy.

There could be some selection bias in this study as neither the three sites nor the respondents were randomly selected. However, each of the three sites is representative of most of the different types of contexts seen across South Africa, and a relatively large number of respondents with a range of different characteristics were interviewed for this study.

The relatively poor acknowledgement, by senior politicians, state authorities, widely publicized 'experts' and members of the community, of the positive effects of antiretroviral drugs and the concomitant lack of excitement among those not already on treatment may impair both individual adherence to treatment and the possible HIV prevention benefits of antiretroviral treatment. A more explicit communication strategy that promotes the benefits of antiretroviral therapy is required. If antiretroviral agents are to compete more successfully in the therapeutic continuum, there needs to be explicit recognition of, and further strategies to counter, the attraction of alternative therapies for patients and the systematic promotion these treatments receive, including from professional health workers. 


\section{References}

1. Global HIV Prevention Working Group. HIV Prevention in the era of expanded treatment access. Menlo Park, CA, USA: Kaiser Family Foundation;2004.

2. Campbell C, Foulis $\mathrm{CA}$, Maimane S, Sibiya Z. "I have an evil child at my house": stigma and HIV/AIDS management in a South African community. Am J Public Health 2005; 95 :808- 815 .

3. Shisana O, Rehle T, Simbayi LC, Parker W, Zuma K, Bhana A, et al. South African national HIV prevalence, HIV incidence, behaviour and communication survey, 2005. Cape Town: Human Sciences Research Council;2005.

4. National Department of Health. National HIV and Syphilis Sero-prevalence Survey of Women Attending Public Antenatal Clinics in South Africa - 2004, Summary Report. Pretoria, South Africa: National Department of Health;2005.

aMedical Research Council, Tygerberg, South Africa; ${ }^{b}$ School of Public Health, University of the Western Cape, Cape Town, South Africa; cTulane School of Public Health and Tropical Medicine, New Orleans, USA; and ${ }^{d}$ Health Systems Trust, Durban, South Africa.

Sponsorship: This study was conducted with financial support from the Centers for Disease Control and Prevention, Atlanta, GA, USA which played no role in the design, analysis or write up of the study. 